\title{
HIPK2-induced p53Ser46 phosphorylation activates the KILLER/DR5-mediated caspase-8 extrinsic apoptotic pathway
}

\author{
Cell Death and Differentiation (2007) 14, 1837-1839; doi:10.1038/sj.cdd.4402186; published online 13 July 2007
}

Dear Editor,

The p53 oncosuppressor is the most common altered gene in human cancers, and its regulation in controlling cell cycle and apoptosis is central in the problem of human tumorigenesis. ${ }^{1}$ In response to DNA damage, p53 is activated mostly at the post-translational level by complex series of modifications, including phosphorylation and acetylation, leading to control cell life and death. ${ }^{2,3}$ Apoptosis has recently been suggested to be a major contribution to p53-mediated suppression of tumor formation, and resistance to apoptosis is one of the major hurdles in the treatment of cancer. ${ }^{4}$ The mechanisms by which p53 accomplishes its apoptotic function have been studied and involve activation of the mitochondrial pathway, the death receptor pathway, and cleavage of downstream caspases. ${ }^{5}$ Numerous publications have recently described the importance of p53 transcriptional regulation of components of both extrinsic and intrinsic apoptotic pathway including Bax, p53AIP1, Noxa, PUMA, Fas/Apo1, KILLER/ DR5, and PERP, although cells that have engaged p53dependent apoptosis typically follow the intrinsic cell death pathway. ${ }^{5}$ Several genes may be recruited simultaneously by p53 within the same cell, probably acting additively or synergistically, whereas others may be more cell typerestricted with regard to their requirement for p53-mediated apoptosis. Either way, it has been suggested that the choice of p53 between life and death may be dictated by its ability to switch on preferentially particular subsets of genes. The molecular basis for the different activation of particular subsets of target genes is not fully understood. Most certainly, multiple molecular mechanisms contribute to p53's target selectivity. There is now ample evidence that covalent modifications on $\mathrm{p53}$, possibly through conformational changes, which alter directly its DNA-binding specificity, may play a critical role in its target genes preference. However, which phosphorylation sites may be modified after DNA damage to induce the different apoptotic pathways has only recently begun to be addressed.

We have recently shown that homeodomain interacting protein-kinase 2 (HIPK2) specifically phosphorylates p53 at Ser46, thereby activating the p53 apoptotic function following severe DNA damage, including ultraviolet radiation and chemotherapeutic drug treatments. ${ }^{6,7}$ Emerging evidence implicates that p53 phosphorylation at Ser46 is a necessary step for inducing irreversible apoptosis in response to severe DNA damage. ${ }^{8}$ This finding has revealed a role for HIPK2 in the post-translational modifications and selective regulation of p53 transcriptional activity. Thus, it has been shown that p53Ser46 phosphorylation is associated with transcriptional activation of proapoptotic mitochondrial p53AIP1 gene, involving the intrinsic apoptotic pathway. ${ }^{8,9}$ In agreement with these data, it has been recently shown that defect in Ser46 phosphorylation contributes to the acquisition of the p53 resistance in an oral squamous cell carcinoma cell line, ${ }^{10}$ while a mutant active form of p53, p53-46F, induces apoptosis more effectively than wild-type $p 53,{ }^{11}$ strengthening the role for this p53 post-translational modification in selective transcription of apoptotic target genes. Starting from these findings, we wanted to investigate whether HIPK2/p53 complex could induce the activation of extrinsic/death receptor pathway.

We overexpressed wild-type Flag-tagged HIPK2 and its kinase-deficient mutant $\mathrm{K} 221 \mathrm{R}$ in 2008 human ovarian carcinoma cells carrying wild-type p53. Viability assay by Trypan blue exclusion and the long-term colony assay showed that HIPK2 overexpression drastically reduced cell viability and cell survival, respectively, compared to K221R mutant (data not shown). Western blot analysis shows that HIPK2 overexpression in 2008 cells induced apoptotic cell death as evidenced by cleavage of the caspase- 3 substrate PARP mediated by caspase- 8 cleavage (Figure 1a). Caspase activity was then estimated by means of fluorogenic assay showing that HIPK2 overexpression, but not K221R mutant expression, induced caspase- 3 and caspase- 8 activities (Figure 1b). We next evaluated the involvement of HIPK2 in activation of extrinsic/death receptor pathway in a more physiological manner. The 2008 cells, treated with adriamycin (ADR), were subjected to immunoblot analyses to show caspase-8 and PARP cleavages (Supplementary Figure S1a), as well as fluorogenic assay for detection of caspase8 activity (Supplementary Figure S1b). Following depletion of HIPK2, 2008 cells strongly reduced their ability to activate caspase-8 activity (Supplmentary Figure S1b), suggesting that HIPK2 is involved in extrinsic/death receptor pathway activation following drug treatment. To confirm these findings, we cotransfected 2008 cells with HIPK2 and DN-caspase-8 mutant expression vectors. This dominant negative mutant protein specifically inhibits the processing of substrates by wild-type caspase-8 enzyme. As shown in Figure 1c, HIPK2-induced caspase- 8 and -3 activities, as detected by 

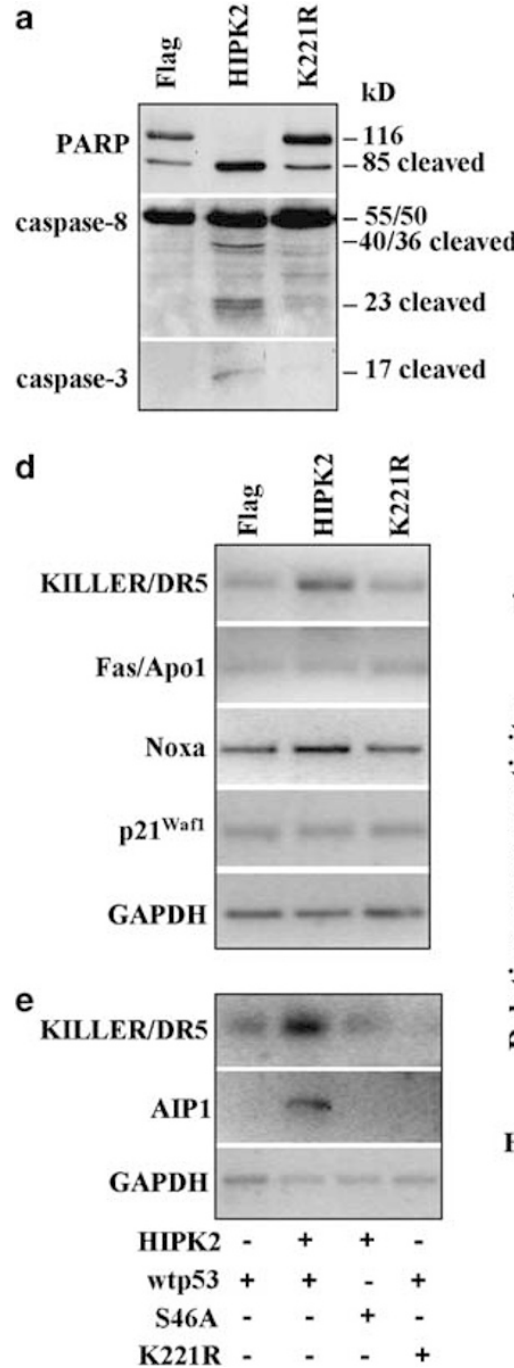

b
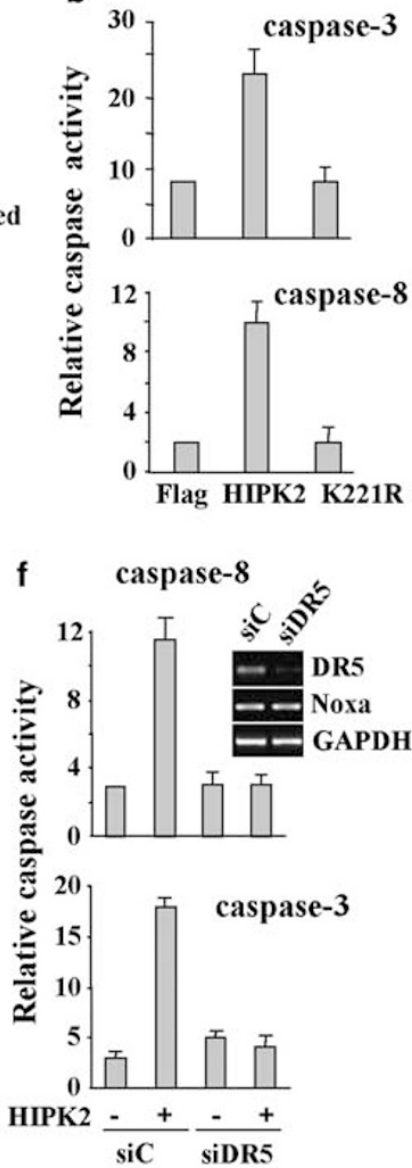

C
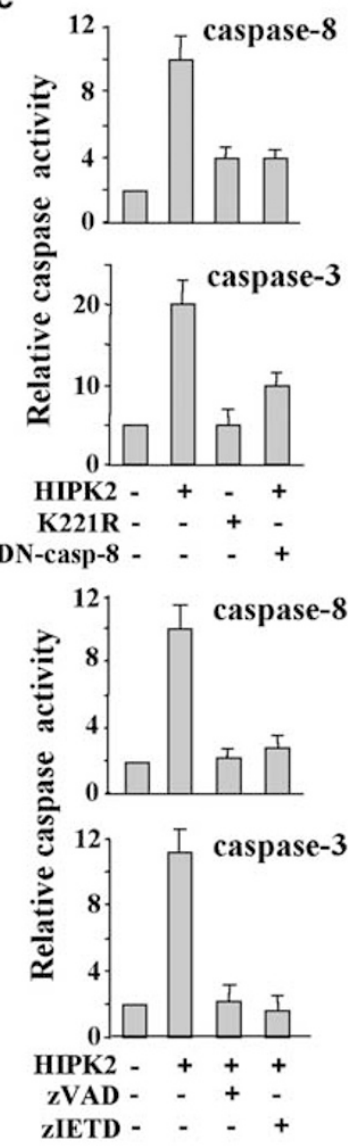

Figure 1 HIPK2/p53Ser46 induces KILLER/DR5-mediated caspase-8 activation. (a) 2008 ovarian tumor cells were transfected with expression vectors encoding wildtype Flag-HIPK2 or Flag-K221R kinase-deficient mutant. Immunoblot of PARP and caspase-8 and -3 cleavages was performed on total cell lysates (TCLs). (b) Caspase3 and -8 activities were evaluated on TCLs by fluorogenic assay following overexpression of 2008 cells with indicated Flag-tagged expression vectors. (c) Caspase-3 and -8 activities were detected by fluorogenic assay following overexpression of 2008 cells with expression vectors encoding HIPK2, K221R, and DN-caspase-8 expression vectors (upper two panels). HIPK2-mediated caspases involvement was evaluated by treating cells with irreversible caspase inhibitors zVADfmk (for all caspases) and ZIETDfmk (for caspase-8), at a concentration of $40 \mu \mathrm{M}$ for each inhibitor: caspase-8 and -3 activities were detected by fluorogenic assay (lower two panels). (d) 2008 cells were transfected with HIPK2 and K221R expression vectors, and p53-induced target genes analyzed for mRNA expression levels. GAPDH was used as control. (e) Apoptotic p53 target genes were anlayzed by RT-PCR in H1299 p53/null cells after coexpression of HIPK2 and K221R in combination with wt-p53 and p53S46 mutant. (f) HIPK2-induced caspase-8 and -3 activities in 2008 cells were detected by fluorogenic assay following impairment of DR5 function by siRNA (RT-PCR analysis in the inset) and subsequent overexpression of HIPK2

fluorogenic assay, were strongly suppressed by DN caspase8 coexpression. We next examined the impact of irreversible caspase inhibitors zVADfmk (for all caspases) and zIETDfmk (for caspase-8) on HIPK2-mediated caspases' activation. The general caspase inhibitor zVADfmk completely blocked both caspase- 8 and caspase- 3 cleavage; similarly, the zIETDfmk drastically reduced both caspases activity (Figure 1c). To test the role of caspase-8 on HIPK2-induced cell death, we measured apoptosis by TUNEL staining. We found a reduction of HIPK2-induced apoptosis, following cotransfection with DN-caspase-8 mutant expression vector or treatment with the caspase-8 inhibitor zIETDfmk (Supplementary Figure S2). Altogether, these results strongly suggest that HIPK2-induced caspase-8/extrinsic pathway activation is involved in apoptotic cell death. The finding that inhibition of HIPK2-induced caspase-8 does not completely abrogate apoptosis suggests the existence of additional caspases involved in HIPK2-induced apoptosis.

To get insight into the molecular mechanisms of caspase-8/ extrinsic pathway activation by HIPK2 and the subsequent involvement of p53 transcriptional activity, we analyzed the expression levels of p53 target genes known to induce apoptotic cell death through caspase-8 activation, including KILLER/DR5 and Fas/APO1. ${ }^{5}$ We performed RT-PCR analyses following HIPK2 and K221R vectors overexpression in 2008 cells. We found that the expression levels of KILLER/ DR5 were upregulated following HIPK2 overexpression, while K221R mutant failed to do so (Figure 1d). Interestingly, Fas/ 
APO1 death receptor was not induced, indicating a cell-type specificity for gene transcription and, in agreement with previous results, ${ }^{6}$ p2 $21^{\text {Waf1 }}$ was not upregulated. Furthermore, the involvement of apoptotic intrinsic pathway was shown by induction of Noxa (Figure 1d). These results indicate target genes transcriptional selectivity mediated by HIPK2 and the involvement of HIPK2 catalytic domain in this function. Therefore, to confirm the involvement of HIPK2-induced p53Ser46 phosphorylation in the transcription of KILLER/ DR5 gene, we overexpressed HIPK2 and K221R vectors in combination with wtp53 and S46A-mutant expression vectors in H1299 p53/null cells. We found that KILLER/DR5 expression levels were upregulated when HIPK2 was coexpressed with wtp53, but not with p5346A mutant (Figure 1e). Similarly, K221R vector coexpressed with wtp53 failed to upregulate both KILLER/DR5 gene and the already known p53AIP1 gene. Finally, we found that HIPK2-induced caspase-3 and -8 activities were strongly impaired by inactivating KILLER/DR5 function using siRNA (Figure 1f). As expected, TUNEL assay revealed reduction of HIPK2-induced apoptosis in the absence of KILLER/DR5 (Supplementary Figure S3a and b). Therefore, we tested the involvement of Noxa (intrinsic pathway) in HIPK2-induced relative caspase-3 and -8 activities and apoptosis. We overexpressed HIPK2 in 2008 cells, following abolishment of endogenous Noxa by siRNA and found a strong reduction of caspase-3 activity, while caspase-8 activity was not affected; in agreement siRNA for Noxa function, reduced HIPK2-induced apoptosis of about $38 \%$ (Supplementary Figure S4a and S4b).

Altogether, these data demonstrate, for the first time, that exogenous HIPK2 can induce apoptosis through caspase-8 activation and that HIPK2-induced p53S46 phosphorylation may regulate the expression of KILLER/DR5 gene, involved in caspase-8/extrinsic pathway activation. However, we also show that the mitochondrial intrinsic pathway is induced by HIPK2 through Noxa, likely explaining why inhibition of caspase-8 does not completely abolish HIPK2-induced apoptosis. These are important findings because the initiator caspases, including caspase- 8 and -9 , appear to have some specificity for different types of upstream signals as well as preferred downstream substrate procaspases. Moreover, components of cell apoptotic machinery are frequently altered in cancer and disruption of apoptotic pathways may be involved in tumor formation, regression, and chemoresistance. The fact that HIPK2/p53Ser46 participates in both the extrinsic and the intrinsic pathways of caspases activation supports the idea that there are multiple downstream effectors of p53 involved in cell death and strengthen the role of this specific p53Ser46 post-translational modification in selective activation of apoptotic target genes. Thus, agents that induce phosphorylation of p53 at Ser46 will favor the transcriptional repertoire of p53 towards proapoptotic genes, thereby favoring death.

Acknowledgements. We thank all the people cited in the text for their generous gifts. We also thank Dr. Silva Soddu for critical reading of the manuscript. This work was supported by Associazione Italiana per la Ricerca sul Cancro (AIRC) and Ministero dell'Universita' e Ricerca (Cofin-Miur).

\section{G Pistritto ${ }^{1}, R$ Puca $^{2}, L$ Nardinocchi', A Sacchi and $G D^{\prime}$ Orazi $^{\star, 2,3}$}

1 Department of Neuroscience, University 'Tor Vergata', 00133 Rome, Italy;

2 Department of Experimental Oncology, Molecular Oncogenesis Laboratory, Regina Elena Cancer Institute, 00158 Rome, Italy; and

3 Department of Oncology and Neurosciences, University 'G. d'Annunzio', 66013 Chieti, Italy

* Corresponding author: G D'Orazi, Department of Experimental Oncology, Molecular Oncogenesis Laboratory, Regina Elena Cancer Institute, Via delle Messi d'Oro 156, 00158 Rome, Italy. Tel: + 3906 52662542;

Fax: + 3906 52662505; E-mail: dorazi@ ifo.it

1. Oren M. Cell Death Differ 2003; 10: 431-442.

2. Appella E et al. Eur J Biochem 2001; 268: 2764-2772.

3. Vogelstein B et al. Nature 2000; 408: 307-310.

4. Attardi L et al. Nat Genet 2004; 36: 7-8.

5. Haupt S et al. J Cell Sci 2003; 116: 4077-4085.

6. D'Orazi G et al. Nat Cell Biol 2002; 4: 11-19.

7. Di Stefano V et al. Exp Cell Res 2004; 293: 311-320

8. Oda K et al. Cell 2000; 102: 849-862.

9. Matsuda $\mathrm{K}$ et al. Cancer Res 2002; 62: 2883-2889.

10. Ichwan SJA et al. Oncogene 2006; 25: 1216-1224.

11. Nakamura Y et al. Cancer Sci 2006; 97: 633-641.

\title{
Prothymosin- $\alpha 1$ prevents necrosis and apoptosis following stroke
}

\author{
Cell Death and Differentiation (2007) 14, 1839-1842; doi:10.1038/sj.cdd.4402189; published online 29 June 2007
}

\section{Dear Editor,}

Prothymosin- $\alpha 1$ (ProT $\alpha$ ), a highly acidic nuclear protein of the $\alpha$-thymosin family, has multiple functions both within and outside of the cell. ${ }^{1}$ ProT $\alpha$ plays a cytoprotective role by inhibiting apoptosome formation, ${ }^{2}$ and it has cell proliferative activity as an extracellular signaling molecule. ${ }^{1}$ Recently, we found that ProT $\alpha$ is released upon necrotic stress and protects cells from neuronal death through activation of a putative $\mathrm{G}_{\mathrm{i} / \mathrm{o}}$-coupled receptor coupled to phospholipase $\mathrm{C}$ and protein kinase $\mathrm{C} \beta_{\| 1 \cdot}{ }^{3}$ 